\title{
Handling Electromagnetic Radiation beyond Terahertz using Chromophores to Transition from Visible Light to Petahertz Technology
}

\section{Langhals $\mathrm{H}^{*}$}

LMU University of Munich, Department of Chemistry, Butenandtstr-13, D-81377 Munich, Germany

\begin{abstract}
An increase of the operating frequencies of electromagnetic waves leads from the well-estalished terahertz technology to the visual and reaches petahertz radiation. It is shown that electromagnetic radiation close to petahertz is attractive for technology where knowledge about radio waves can be applied. The dimensions of such radiation are still classically macroscopic; however, molecular components such as resonators were used where quantum mechanics rules have to be considered. Constructions of coupled resonators for energy transfer are as well demonstrated as molecular components for optical metamaterials.
\end{abstract}

Keywords: Terahertz; Petahertz; Electromagnetic radiation; Dyes; Visible light; Resonators; FRET; Energy transfer; Metamaterials; Reflectance

\section{Introduction}

The technology of radio waves representing electromagnetic radiation is well-established and dominated by metallic conductors and electronic circuits where both the radiation and the operating devices are macroscopic (large, classical) [1]. There is a tendency for increasing the operating frequency both because of the increase of the number of possible ports, the increase of information recording and the decrease of the size of antennae and other involved components. One may demand if there is a natural technological limit for the increase in frequency.

\section{Materials and Methods}

The dyes were prepared according to the literature [2]. Pellets were prepared from $150 \mathrm{mg}$ of pigments in a compacting tool with a polished round steel heading die of $13 \mathrm{~mm}$ diameter (compacting tool for $\mathrm{KBr}$ pellet in infrared spectroscopy). The compacting tool was evacuated to $0.2 \mathrm{mBar}$ and 10 tons were applied for $5 \mathrm{~min}$; this corresponds to 7500 Bar. The UV/Vis absorption spectra were recorded in chloroform in 1 $\mathrm{cm}$ cuvettes with extinctions between 0.7 and 1.0 in the maxima. The fluorescence spectra were recorded in chloroform in $1 \mathrm{~cm}$ cuvettes with extinctions between 0.01 and 0.02 in the maxima (Figure 3).

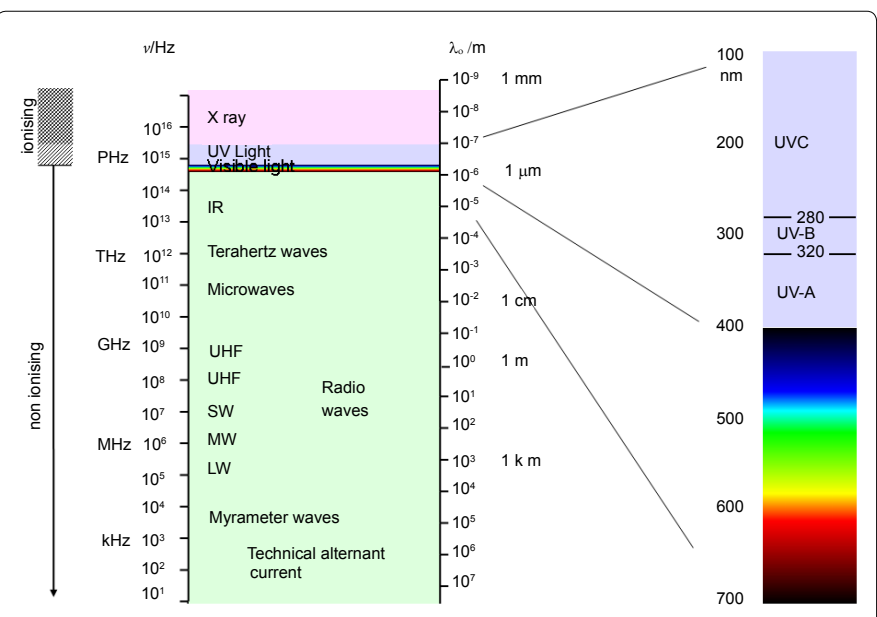

Figure 1: Important ranges of electromagnetic waves. The visible is indicated and enlarged. Ionisation becomes more and more dominating at short wavelengths.

\section{Operating electromagnetic radiation at very high frequencies}

Technologically important ranges of electromagnetic radiation are shown in Figure 1. The frequency $v$ of electromagnetic radiation is interlinked with a wavelength $\lambda$ and the velocity of light $c$ in terms of $\lambda=c / v$. The wavelength in vacuo is reported in Figure 1 according to the velocity $c_{\mathrm{o}}$ of light in vacuo. The index of refraction $n_{v}$ has to be considered for the propagation of electromagnetic waves [3] in matter where $c=c_{\mathrm{o}} / n_{v}$ (a complex index of refraction has to be applied for a complete description, however, this is simplified to $n_{v}$ in this context); correspondingly, the wavelength shrinks to $\lambda / n_{v}$ The index of refraction $n_{v}$ is equal the square root of the relative permittivity $\varepsilon_{r}\left(n_{v}=\sqrt{\varepsilon_{r}}\right)$ for radio frequencies and non-magnetic materials. More and more deviations are observed for very high frequencies and condensed matter. This is why the rearrangement of the atomic nuclei as a response to an external electric field dominates for low frequencies and polar condensed matter. The comparably slow movement of the heavy nuclei cannot follow the electric field for very high frequencies at about $1 \mathrm{PHz}$ where the response of the movement of the electrons becomes the dominating effect because of their lower mass (this is indicated by the polarizability of matter). However, the behaviour becomes more complicated close to absorption bands by the complex index of refraction (anomalous refraction). As a consequence, the polarizability should be measured by means of the index of refraction well separated from absorption bands. The centre of the visible at about half a micron in vacuum wavelength is a good compromise therefore because it is well situated for the majority of materials between strong absorption band in the UV and in the NIR. The position of the sodium double D spectral line at 589/590 nm was taken as the reference wavelengths for the determination of the indices of refraction at $20^{\circ} \mathrm{C}\left(n_{\mathrm{D}}{ }^{20}\right)$ for historical reasons and is still a good compromise for reference measurements.

$$
E=h \cdot v=h \cdot c / \lambda
$$

*Corresponding author: Langhals H, LMU University of Munich, Department of Chemistry, Butenandtstr-13,D-81377 Munich, Germany, Tel: +49 892180 77699; E-mail: Langhals@Irz.uni-muenchen.de

Received February 01, 2014; Accepted April 16, 2014; Published April 26, 2014

Citation: Langhals H (2014) Handling Electromagnetic Radiation beyond Terahertz using Chromophores to Transition from Visible Light to Petahertz Technology. J Electr Electron Syst 3: 125. doi:10.4172/2332-0796.1000125

Copyright: @ 2014 Langhals H. This is an open-access article distributed under the terms of the Creative Commons Attribution License, which permits unrestricted use, distribution, and reproduction in any medium, provided the original author and source are credited. 
Electromagnetic radiation consists of individual quanta where their frequencies and wavelengths, respectively, are related to the energy $E$ according to Einstein's formula (1) where $h$ is Planck's constant. The individual quanta are of minor importance for radio transmitters because of their low energy; sometimes consequences of the quantization are observed in the amplification low-level signals. However, the energy of the individual quanta increases for higher frequencies, finally reaches the energy of the chemical bonds in technical materials, can cleave the bonds and can cause damage of the material. Such radiation is named ionizing radiation. The energy in chemical reactions is commonly reported for a mole of material. As a consequence, equation (1) for individual quanta has to be multiplied with Avogadro's number to obtain equation (2a) and (2b), respectively, for a general purpose comparing with other chemical processes.

\section{$E=28591 \mathrm{kcal} \cdot \mathrm{mol}^{-1} / \lambda$}

\section{$E=119700 \mathrm{~kJ} \cdot \mathrm{mol}^{-1} / \lambda$}

The energy of quanta reaches the energy of standard chemical bonds at about $1 \mathrm{PHz}(1000 \mathrm{THz})$ in the visible and in the UV; see Figure 1. However, there is no strict limit because very special chemical structures allow radiation-induced chemical processes even in the NIR close to the visible and find applications in photographical infrared films [4]. With increasing frequencies, more and more structures become suitable for radiation-induced processes in the visible and even more in the UV until becoming ubiquitary by X-rays. This causes a damage of materials by ionization and propagates with increasing doses of radiation. As a consequence, the visible becomes attractive for advanced technology of electromagnetic radiation because of the high frequencies of operation and the possibility of controlling the stability of setups by the construction of suitable chemical structures. Moreover, more labile chemical structure may be integrated as an interface to other physical effect such as the construction of sensors [5].

\section{Devices}

The very high frequencies of a technology at about $1 \mathrm{PHz}$ require fundamental alterations of the concept for the components of devices. The technology of radio frequencies is dominated by metallic conductors for antennae, wave-propagating lines, resonating circuits and macroscopic (large, classical) switching devices such as transistors [6]. The short wavelength of visible radiation of about half a micron requires $\lambda / 4$ antennae with lengths of slightly more than $100 \mathrm{~nm}$ and the structure-giving dimensions should be smaller by two powers of ten or even more. Such requirements are beyond the scope of the present nanotechnology by far. Extensions of one nm or less mean the dimensions of molecular structures. Moreover, such small dimensions cannot be altered continuously as in macroscopic, classical structures but only stepwise because of limitations by the extension of chemical bonds such as about $0.15 \mathrm{~nm}$ for carbon-carbon bonds.

The molecular approach to petahertz devices would be an alternative where complex chemical structures can be constructed by well-established chemical synthesis (bottom-up approximation instead top-down approximation). Organic molecules are the most attractive structures for such purposes because they can form stable complex three dimensional frameworks with well-defined rigid structures as a $\sigma$-skeleton formed by localized covalent centrosymmetric C-C-bonds. Electronic functions can be introduced into the $\sigma$-skeleton by additional $\pi$-bonds forming double bond structures [7] where the alternation of singles and double bonds (conjugated systems) allows shifting of electrons as is known for metallic conductors and can be applied as antennae for electromagnetic radiation. Generally, the dimensions of preparatively efficiently accessible and well-defined operating structures of that type extend between 1 and $3 \mathrm{~nm}$ where the lengths of such antennae are generally too short with respect to the wavelengths of the interacting radiation and have to be considered for construction and operating. Classical, macroscopic resonating circuits are realized by means of capacitors and coils for radio frequencies or lower [6]. This is difficult to realize for molecular structures because of the restricted variation of structure limited by the arrangements of atoms. Electronic transitions between molecular eigenvalues, molecular energy levels, can be an alternative; these are given by the special chemical structure of the applied molecules. This concept leads to the situation where the electromagnetic radiation of wavelengths of half a micron is still macroscopic (large, classical), whereas both the antennae and the resonators are microscopic (small) in dimensions where physical effects are dominated by quantum mechanic rules.

Chemical structures absorbing visible light are well established in organic dyes [8]. Their chromophores (light-absorbing structures) may be adapted to the special requirements for components of petahertz technology. The chemical and photochemical stability of such materials is of central importance for the realization of long-term operating devices where extended aromatic structures [9] combine both requirements, a solid $\sigma$-framework (firm, localized covalent bonds) and $\pi$-bonds for operating, both stabilized by aromatism. Strongly fluorescent structures where the absorbed light is re-emitted are preferred as components because there is no leakage of the absorbed energy until re-emission caused by the natural transition probability. Suitable and attractive chromophores of this kind are the peri-arylenes [Figure 2] [2,10-12].

\section{Chromophores: peri-Arylenes}

Figure 2 indicates the general chemical structure of peri-arylenes [2]. The light absorption is strongly influenced by the length of the chromophore where the absorption for $n=1$ is in the UV, for $n=2$ in the middle of the visible, for $n=3$ in the region of the visible at long wavelengths and extends for $n=4$ until the NIR; see Figure 3 .

The thermal, chemical and photochemical stability of the periarylenes is extraordinarily high and means a good prerequisite for the construction and application of complex functional structures. The purely organic components render recycling uncritical and make such materials suitable for industrial mass production. Moreover, strong fluorescence is observed for homologous with $n>1$ and means that the energy of the absorbed radiation remains on the chromophores until natural decay with a lifetime of about 4 ns proceeds by emission of fluorescence light. The substituents $\mathrm{R}$ in Figure 2 are decoupled<smiles></smiles>

Figure 2: peri-arylenes 1. 


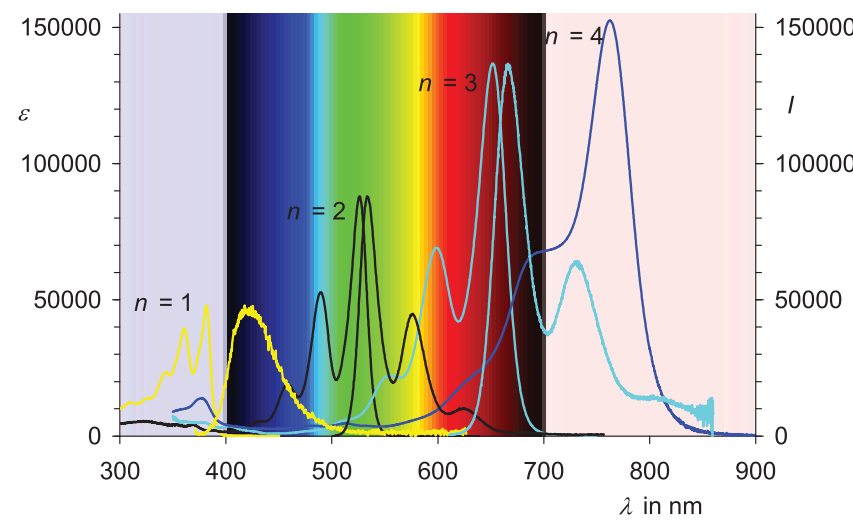

Figure 3: UV/Vis/NIR absorption (left, left scale with molar absorptivities $\varepsilon)$ and fluorescence (right, right scale with arbitrary units $l$ ) spectra of periarylene dyes 1 in homogeneous solution in chloroform. From left to right: Naphthalene ( $n=1$, yellow), perylene ( $n=2$, black), terrylene $(n=3$, turquoise) and quaterrylene ( $n=4$, blue) carboximides.

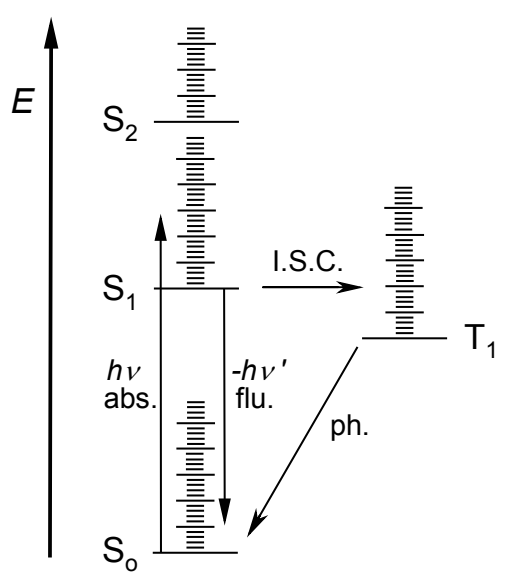

Figure 4: Jablonski's diagram for absorption (abs.) of the electromagnetic energy $h v$ and emission of light by dyes in the scale of the energy $E . \mathrm{S}_{0}, \mathrm{~S}_{1}$ and $S_{2}$ : Molecular electronic singlet states of the dye, $T_{1}$ : Electronic triplet state, flu: Fluorescence of the energy $\left(-h v^{\prime}\right)$, ph.: Phosphorescence, I.S.C.: Inter system crossing from the singlet to the triplet state. Long lines: Basic electronic states, medium long lines: Vibration-excited states, short lines: Rotation-excited states in the gas phase and vibrations of libration in the condensed phase, respectively.

from the light-absorbing process by nodes in the orbitals (segment for the population by electrons) responsible for light absorption. As a consequence, these positions can be used for the linking to other chromophores or to operating structures without interfering the optical properties of $\mathbf{1}$. Long-chain sec-alkyl substituent R (swallow-tail substituents such as 1-hexylheptyl) renders such materials soluble [13]. The absorption spectra of $\mathbf{1}$ can be tuned by means of substituents at the aromatic nuclei and thus, can be adapted to special requirements of the operating structure [2].

\section{Absorption, fluorescence and phosphorescence}

The electronic systems of chromophores such as $\mathbf{1}$ form distinct allowed molecular energy levels such as $\mathrm{S}_{0}, \mathrm{~S}_{1}, \mathrm{~S}_{2}$ and so on and are known as eigenvalues determined by quantum mechanics; see Figure 4 (these terms may be differently used in other subjects such as solidstate physics). The energy of radiation according to formula (1) can be resonantly transferred to chromophores inducing electronic transitions between eigenvalues such as between $\mathrm{S}_{\mathrm{o}}$ and $\mathrm{S}_{1}$. At room temperature, the dye molecules remain in the electronic ground state $S$ because the upper molecular electronically excited states such as $S_{1}$ cannot be thermally reached. There are also vibration levels of the chromophores (medium long lines in Figure 4) forming more dense ladders of states beginning at the individual electronic states. Finally, there are even more densely packed states of rotational levels in the gas phase and less well defined and overlapping vibrations of libration in the liquid phase (small lines in Figure 4). The latter form quasi continua rather than individual lines being responsible for the continuous spectra of absorption and fluorescence in Figure 3.

Electronic transitions with absorption of electromagnetic waves at room temperature ( $h v$ abs. in Figure 4 ) start at the basic electronic $\mathrm{S}$ state and reach electronically excited states with possibly excitation of vibration and vibration of libration where there is a limit at low energy (low frequency and short wavelengths, respectively) determined by the energetic difference between the $S_{0}$ and $S_{1}$ ground states; this corresponds to the band gap in semiconductors. The degrees of freedom both of vibration and vibration of libration and even of further electronic excitation are strongly coupled with the electronic excitation; as a consequence, a fast relaxation with the dissipation of the excess energy proceeds within femtoseconds to the $S_{1}$ ground state; the latter exhibits a longer lifetime of commonly several nanoseconds. The $S_{1}$ ground state is the starting point for further processes such as photochemistry and fluorescence ( $-h v^{\prime}$ flu. in Figure 4). Electrons are Fermi-type particles where each energetic level can be occupied by maximal two electrons with antiparallel spin resulting in the singlet $S_{\text {o }}$ ground state reported in Figure 4. The electrons of the highest occupied orbital were re-located in two different orbitals as a consequence of the electronic excitation so that two realizations, the $S_{1}$ and the $T_{1}$ state, become possible where the electron spins of the latter are parallel. An allowed photo-induced transition requires the retaining of the spin so that the $S_{1}$ state will be reached. Relaxation processes (I.S.C., inter system crossing in Figure 4) allow the formation of the energetically lower lying and longer living $\mathrm{T}_{1}$ state [14] where phosphorescence (ph. in Figure 4) may occur as light-emission. The similar energetic ladders of vibronic states in $S_{0}$ and $S_{1}$ causes a mirror-type structuring of the absorption and fluorescence spectra; see Figure 3. However, this mirrortype is not perfectly realized where the intensities of the higher vibronic bands are relatively more intense in absorption than in fluorescence. This is a consequence of the mismatch between the small molecular dimensions of about $1 \mathrm{~nm}$ as antennae with the dimensions of the wavelengths of light of about $500 \mathrm{~nm}$. This is even more pronounced in fluorescence than in the absorption because of longer wavelengths. A quantitative relation between lengths and efficiency of short antennae is given by Rüdenberg's equation [15-17] where the efficiency given by the radiation resistance is proportional to the square of quotient of the effective lengths of a radio antenna over the wavelengths and finds in counterpart in the similar Ross' equation [18] in quantum chemistry.

\section{Line shape of UV/Vis spectras}

A single relaxation process would lead to an exponential damping of the propagating electromagnetic wave in the domain of time; this would result in a Lorentzian line shape in the frequency domain (see Fourier transformation); such line shape is known for simple resonating circuits. However, multi relaxation processes are possible concerning the interaction of chromophores with UV/Vis radiation. As a consequence, a Gaussian line shape is expected such as is given in equation (3) where $E_{\max }$ is the absorpivity at the maximum of the band and $E_{v, \lambda}$ at the individual frequency $v$ and the wavelength $\lambda$, respectively. $v_{\max }$ and $\lambda_{\max }$, respectively, are the positions of maximum of the band. $\sigma$ 


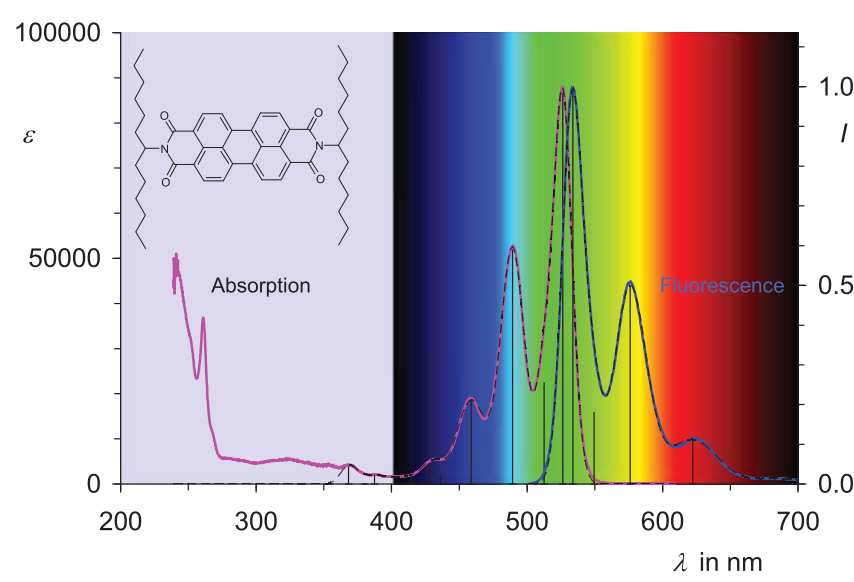

Figure 5: Absorption (magenta curve, $\varepsilon$ on the left side) and fluorescence (blue curve, normalized intensity $I$ on the right side) spectra of $1(n=2, \mathrm{R}=1$. hexylheptyl) in chloroform. Black-dashed lines: Simulated spectra on the basis of a Gaussian analysis according to equation (3); Bars: Positions and intensities of the individual Gaussian bands for $\lambda>350 \mathrm{~nm}$.

is the standard deviation and characterizes the extension of the bands. The compatibility of equation (3) with experimental data was tested $[19,20]$ where a transformation according the right part of equation (3) is recommended [21] because UV/Vis spectra are commonly linearly recorded in wavelengths. The factor 100 in the exponent is useful because the wavelength is commonly reported in $\mathrm{nm}$ and extensions of bands such as $\sigma$ in $\mathrm{kK}\left(10000 \mathrm{~cm}^{-1}\right)$ (Figure 5).

$$
E_{\nu, \lambda}=E_{\max } \cdot e^{\frac{\left(v-v_{\max }\right)^{2}}{2 \sigma^{2}}}=E_{\max } \cdot e^{\frac{100 \cdot\left(\frac{1}{\lambda}-\frac{1}{\lambda_{\max }}\right)^{2}}{2 \sigma^{2}}}
$$

Typical structured absorption and fluorescence spectra were found for $\mathbf{1}$ ( $n=2, \mathrm{R}=1$-hexylheptyl) in homogeneous solution in chloroform; see Figure 5 . The spectra could be split into individual Gaussian bands according to equation (3) as is shown in Figure $5\left(E_{v, \lambda}\right.$ and $E_{\max }$ have to be replaced by $I_{v, \lambda}$ and $I_{\max }$ for fluorescence). The re-calculated spectra on the basis of these bands perfectly fit the experimental spectra (see dashed black curves in Figure 5). The Gaussian line-shape characterizes more generally the absorption and fluorescence bands of dyes as is shown with many examples [21].

\section{Absorption and reflectance}

The common linear optical behaviour of matter as a function of time is described by equation (4) [2], where $E$ is the electrical field and $P$ the resulting polarization.

$$
\mu \cdot P^{\mu}+f \cdot P+D \cdot P=N \cdot q^{2} \cdot E
$$

$\mu$ is a measure for inertia, $f$ for friction, and $D$ for elasticity concerning the applied field, where as $N \cdot q^{2}$ characterizes the interacting charge. Equation (4) can be simplified for the response of a single light quantum when $E=0$ and by setting the quotient $f / \mu$ to $1 / \tau$, the reciprocal of a characteristic lifetime and $D / \mu$ to $\omega^{2}$, the square of a characteristic frequency, the plasma frequency; thus, equation (5) results.

$$
P+\frac{P}{\tau}+\omega^{2} \cdot P=0
$$

The solution of equation (5) is given by equation (6) where $A_{1}$ and $A_{2}$ are the two constants of integration and $t$ is the time.

$$
P=e^{-\frac{t}{2 r}} \cdot\left(A^{1} \cdot e^{\sqrt{\frac{1}{4 \tau^{2}-\omega^{2} \cdot t}}}+A_{2} \cdot e^{-\sqrt{\frac{1}{4 \tau^{2}}-\omega^{2} \cdot t}}\right)
$$

The radicands of the square roots in equation (5) are negative for the majority of materials in the visible because of the very high frequency $\omega$ of about $1 \mathrm{PHz}(1000 \mathrm{THz})$. Thus, the exponents become imaginary and represent a propagating wave for the underdamped solution (oscillatory solution) of equation (4). The first exponential factor in equation (6) represents the damping of the wave and corresponds to the damping according to Lambert Beer's law where the light flux $\phi$ is proportional to the square of the electric field respectively.

$$
E=\varepsilon \cdot c \cdot d
$$

An increase of absorptivity and a concomitant increase in $1 / \tau$ and $1 / \tau^{2}$, respectively, lead finally to the critically damped solution of equation (5) because the square roots in equation (6) become zero or even positive for a further increase. As a consequence, there is no propagating wave in the material and the reflection of the wave becomes dominant; compare ref. [22-24]. For this limiting behaviour, the pre-exponential damping factor can be compared with absorptivity $E$ (a confusion between $E$ and the electrical field $E$ should be avoided) as the damping according to Lambert-Beer's law (7) where $\varepsilon$ is the molar absorptivity, $c$ the chemical concentration of chromophores and $d$ the path lengths of the propagating wave corresponding to $t$ in (6). A critical molar absorptivity $\varepsilon$ for a pure dye is defined by equation (8) where $n_{v}$ is the real component of the index of refraction, $M_{n}$ the molecular weight, $\lambda_{\mathrm{o}}$ is the vacuum wavelength and $\rho_{d}$ the density of the dye.

$$
\varepsilon=\frac{4 \pi \cdot n_{v} \cdot M_{n}}{\lambda_{o} \cdot \rho_{d} \cdot \ln 10}
$$

This limit might be reached by highly absorbing organic dyes $[25,26]$. The perylene tetracarboxylic bisimides $(1, n=2)[11,12]$ and the anhydride $\mathbf{2}$ are well-known for their high molar absorptivities and light-fastness and are therefore suitable for testing the application of equation (6) and (8), respectively (Figure 6).

The anhydride 2 exhibits a density of $1.84 \mathrm{~g} \cdot \mathrm{cm}^{-3}$ as a solid and a molecular weight of 674 . The critical absorptivity according to equation (6) is obviously not reached for the solid powder and thus, 2 is generally employed as a red pigment selectively absorbing light; see Figure $7 \mathrm{a}$ (Figure 7).

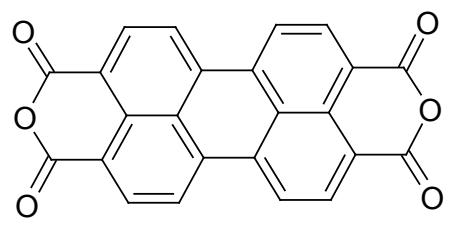

Figure 6: perylene tetracarboxylic bisimides anhydride 2.

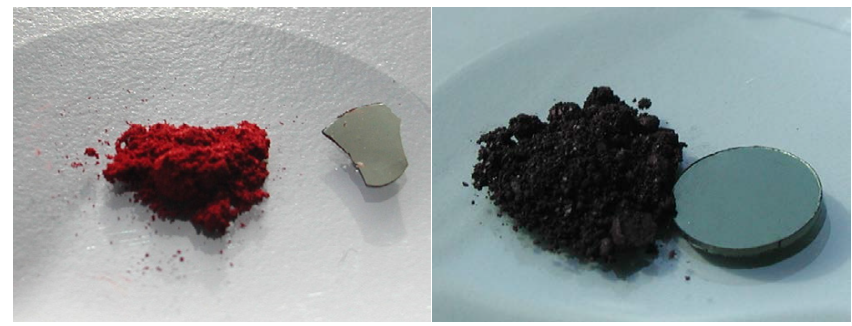

Figure 7: Left (a) pigment 2: Red powder in the left and a golden shiny plate in the right after the application of pressure ( 7500 bar with evacuation) by means of a polished piston. Right (b) pigment $1(n=2, \mathrm{R}=\mathrm{H})$ : black powder in the left and metallic plate after the application of pressure. 
However, the density and packing of 2 can be increased by the application of pressure with 7500 bar (110000 psi) with evacuation to obtain a metallic golden shiny material. A golden shiny mirror-like plate was obtained with a polished press die; see Figure 7a. Obviously, the critical damping according to equation (4) could be exceeded with the higher density and packing. The increase of density by the application of pressure could be verified with other dyes such as for the perylene bisimide $(1, n=2, \mathrm{R}=\mathrm{H}$, pigment red 179$)$, see Figure $7 \mathrm{~b}$ for the pigment powder and the compressed material with a more dark bluish metallic lustre, and the DPP dye irgazin red 254. Nearly no effects are observed for malachite green and for crystal violet because the chromophores are highly light-absorbing and the pure crystals seem to be already so densely packed that they form metallic shining solids.

The opposite effect can be obtained with solid metallic gold forming a mirror-like reflecting material because of the high absorptivity of gold for visible light. A dilution of gold by silicate forms the well-known gold ruby glass selectively absorbing light with no reflection.

\section{Interacting of chromophores}

A single chromophore acts as a single resonator for electromagnetic waves. A complex behaviour is observed for interacting chromophores where two identical chromophores cause a splitting of the initial band (black curves in Figure 8, upper left) into two novel bands at shorter and longer wavelengths known as the Davydov splitting $[27,28]$ by exciton interactions. The intensities of the absorption bands depend on the orientation of the transition dipoles schematically indicated as thick, black lines in Figure 8.

Absorption at long wavelengths become favoured for collinear arrangements of dipoles and the transition at shorter wavelengths suppressed (red line in Figure 8 upper right). On the other hand, a coplanar arrangement suppresses the transition at long wavelengths and favours the transition at short wavelengths (blue line in Figure 8 lower left). Finally, a skew-type arrangement allows both transitions (Figure 8 right, bottom) where the relative intensities and positions depend on the exact geometry. An orthogonal arrangement extinguishes the exciton interactions; this could be demonstrated with a trichromophoric dye [29] where a Cartesian-named backbone [30] places the three chromophores orthogonally into the three room directions.

\section{Resonance energy transfer}

The interaction of two different chromophores allows the establishment of an energetic gradient where the chromophore operating at shorter wavelengths (the donor D) allows the absorption of electromagnetic radiation with higher energy. The subsequent resonance energy transfer as a consequence of dipole dipole interactions transfers the absorbed energy between the components. The fast thermal dissipation of excess vibronic energy causes that the absorbed energy finally is set on the chromophore with the lower energy between the $S_{1}$ and $S_{0}$ named the acceptor (A). The energy transfer was firstly [31] described as a consequence of a resonant interaction of the dipoles of the energy donor D and the energy acceptor A by Perrin [32]. Förster was able to develop Formula (9) for the quantitative description [3336 ] of such a process where $k_{F R E T}$ is the rate constant for the process; such processes are generally named FRET (Förster Resonant Energy Transfer)

$$
k_{F R E T}=\frac{1000 \cdot(\ln 10) \cdot \kappa^{2} \cdot J_{D A} \cdot \phi_{D}}{128 \cdot \pi^{5} \cdot N_{A} \cdot \tau_{D} \cdot\left|R_{D A}\right|^{6}}
$$
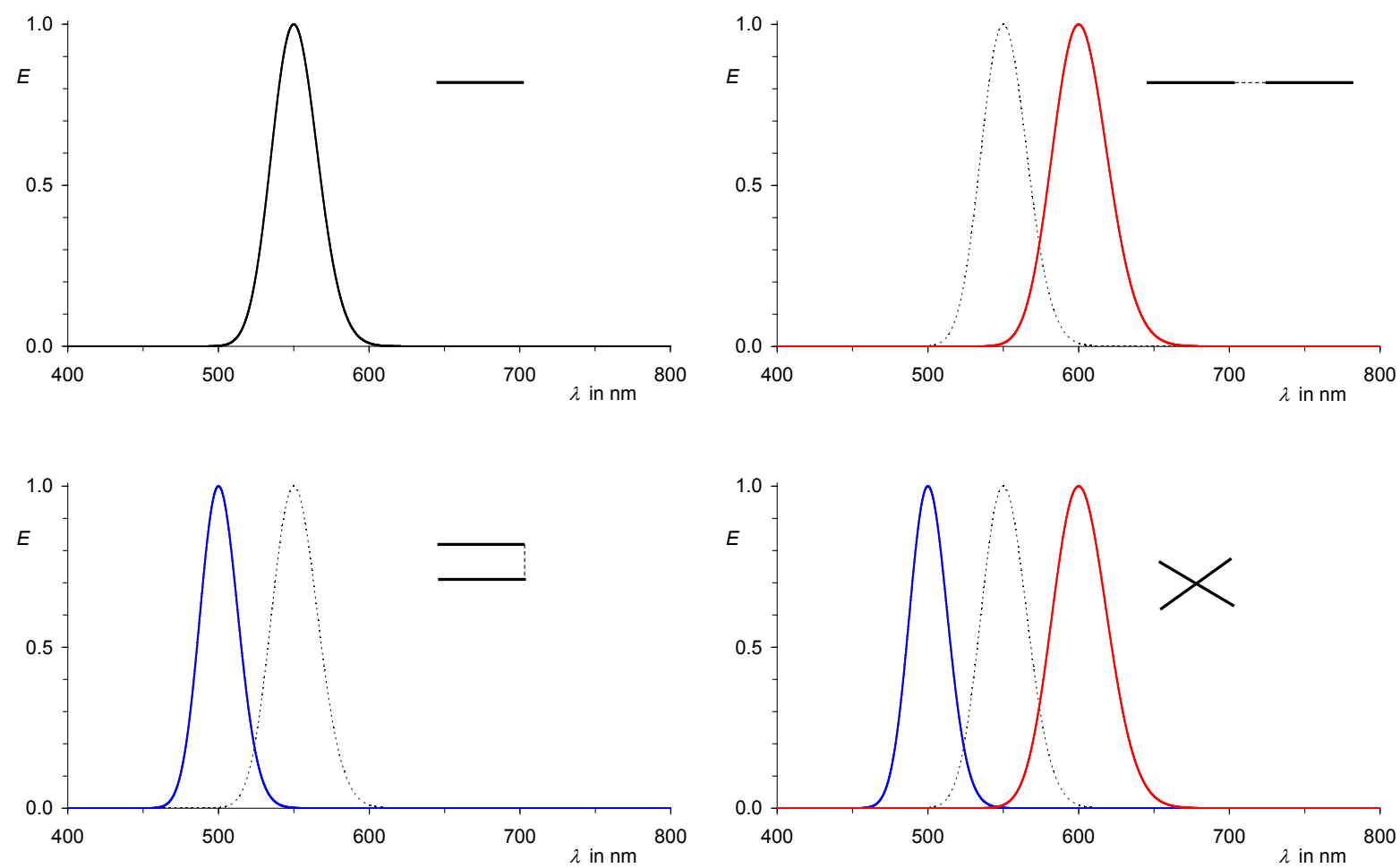

Figure 8: Schematic spectra of two interacting chromophores. Upper left, black curve: Schematic individual spectrum. Upper right: Linearly arranged transition moments favour the absorption at longer wavelengths (red curve) compared with the initial spectrum (black dotted curve). Lower left: Coplanar arranged transition moments favour the absorption at short wavelengths (blue curve) compared with the initial spectrum (black dotted curve). Lower right: Skew-type arrangements of the transition moments allow both transitions (blue and red curves) compared with the initial spectrum (black dotted curve). 


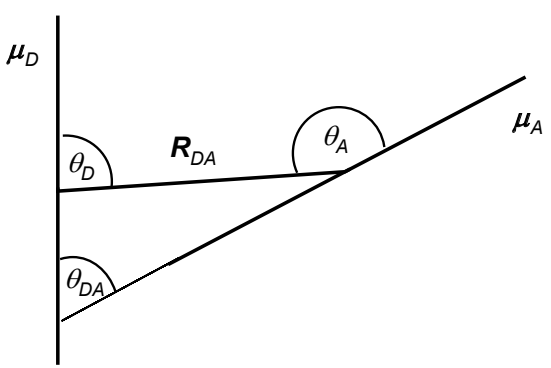

Figure 9: Orientation of the transition dipoles of the energy donor $\boldsymbol{\mu}_{D}$ and acceptor $\boldsymbol{\mu}_{A}$ and the interconnecting vector $\boldsymbol{R}_{D A}$ (projection into the plane).

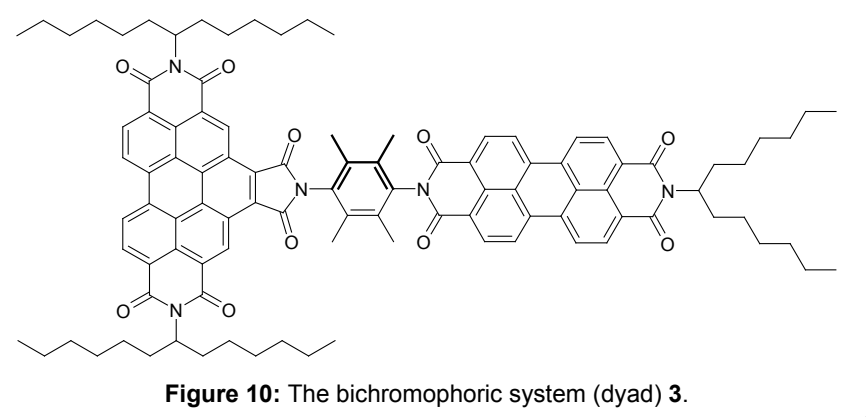

There are mathematical terms and natural constants such as Avogadro's number $\left(N_{A}\right)$ in equation (9) as well as the overlap integral $J_{D A v}$ between the fluorescence spectrum of the energy donor (D) and the absorption spectrum of the acceptor (A). There are two chemically important physical variables, the distance $R$ between the middle points of the transition dipoles (see Figure 9) and the orientation factor $\kappa$. The dependence of the rate constant with the sixth power of the distance allows as well an efficient controlling of the process as the determination of distances and changes of distances in molecular dimensions such as with a molecular ruler (Figure 9).

$$
\begin{aligned}
& \kappa=\left(\hat{\mu}_{D} \cdot \hat{\mu}_{A}\right)-3\left(\hat{\mu}_{D} \cdot \hat{R}_{D A}\right) \cdot\left(\hat{R}_{D A} \cdot \hat{\mu}_{A}\right) \\
& \kappa=\cos \left(\theta_{D A}\right)-3\left(\cos \left(\theta_{D}\right) \cdot \cos \left(\theta_{A}\right)\right)
\end{aligned}
$$

The orientation factor $\kappa$ is formed by a sum of scalar products of the transition dipole moments and the interconnecting vector according to equation (10) where $\kappa^{2}=2 / 3$ is obtained for statistical orientation. Equation (10) can be simplified to the arrangement of cos functions in equation (11); for the angles in (11) see Figure 7. The orientation factor $\kappa$ should become zero for orthogonally arranged transition dipoles if the interconnecting vector of the middle points of the dipoles is also orthogonal to one of the vectors where the energy transfer is expected to be extinguished (Figure 10).

The bichromophoric system (dyad) 3 was synthesized [37] for a test where the orthogonal arrangement according to equation (11) was fulfilled because the transition moment are parallel to an N-Nconnection line both for the left chromophore as the energy donor (D) and the right chromophore as the energy acceptor (A). An extinguishing of the energy transfer is expected according to equation (11), however, a very fast (9.4 ps) and efficient $(\approx 100 \%)$ energy transfer was observed for $\mathbf{3}$ and similar molecules [38,39]. More recent results indicate that the dependence of the rate constant $k_{F R E T}$ on the distance $R$ is better described with an $R^{-3}$ dependence for proximate chromophores and alters to an $R^{-6}$ dependence for more distant chromophores [40].
The dipole dipole interactions seem to be important for the energy transfer, however, they are not the exclusive process and molecular dynamics seem to be equally important. This has to be considered for the construction of complex interacting chromophoric systems. On the other hand, the energy transfer allows the construction of controlled molecular pathways such as in conventional electronics.

\section{Molecular metamaterials}

The electric component $\boldsymbol{\mu}_{\mathrm{e}}$ is dominating in molecular optical transitions. The introduction of a magnetic component $\boldsymbol{\mu}_{\mathrm{m}}$ may introduce non-conventional optical effects. An orthogonal arrangement of $\boldsymbol{\mu}_{\mathrm{e}}$ and $\boldsymbol{\mu}_{\mathrm{m}}$ is of special interest because molecular components for optical metamaterials are obtained where negative indices of refraction can be established [41]. Such metamaterials are popular because of the invisible cloak (magic cap, magic cloak), however, also may be important for the construction of very thin lenses and other advanced optical components. Metamaterials were constructed for microwaves with split ring resonators [42,43], however, a molecular realization of such a component is difficult. On the other hand, metamaterials can be established by means of two parallel resonators for the electric component $\mu_{\mathrm{e}}$ where the magnetic component $\boldsymbol{\mu}_{\mathrm{m}}$ will be formed by the dielectric displacement current in between; see Figure 11, (Figure 12).

An arrangement according to Figure 11a was realized on a molecular scale in 4 [44] where the terminal chromophores represent the electric conductors because of their pure electric transition moments polarized

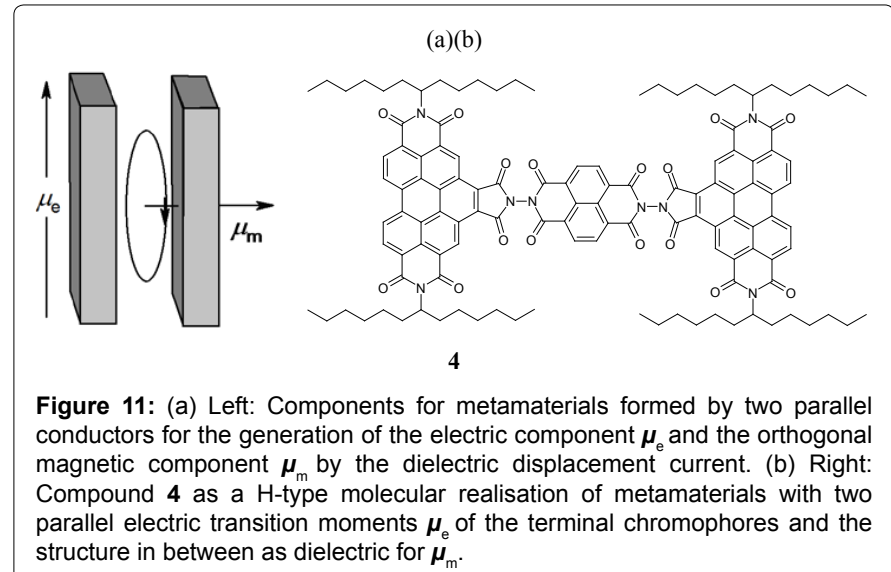
structure in between as dielectric for $\boldsymbol{\mu}_{\mathrm{m}}$

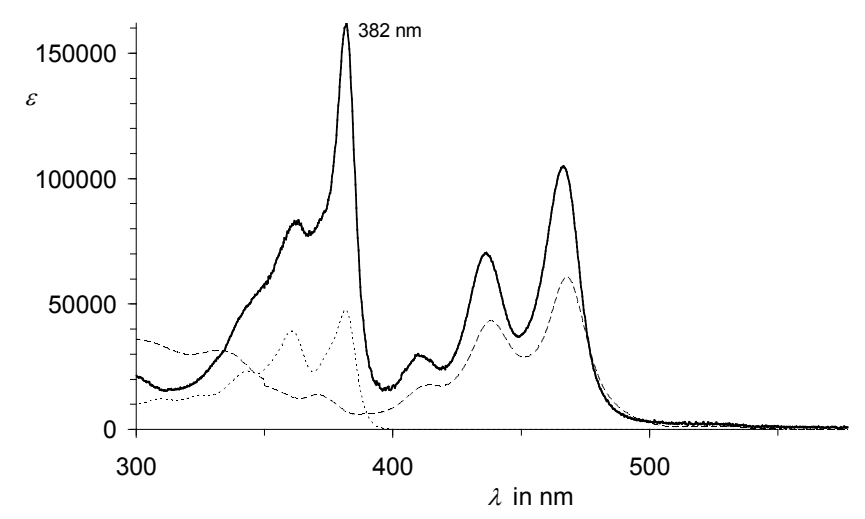

Figure 12: UV/Vis absorption spectrum of 4 in chloroform (solid line) compared with the spectra of the termini (dashed line) and the dielectric linker in between (dotted line). 
along their long axis. The interlinking structure in the middle of the $\mathrm{H}$ forms the dielectric (it absorbs in the UV). The UV/Vis spectrum of 4 mainly consists of an addition of the spectra of the components except one sharp, intense additional absorption at $382 \mathrm{~nm}$; this is attributed to the special $\mathrm{H}$-type meta arrangement of the chromophores in $\mathbf{4}$. As a consequence, molecular components for metamaterials can be constructed by special arrangements of chromophores. A special pattern of these molecular components may form bulk optical metamaterials; the application of liquid crystals is an attractive way therefore.

\section{Conclusions}

A continuation of terahertz electromagnetic radiation to higher frequencies and shorter wavelengths, respectively, passes the visible and reaches 1 petahertz in the UVB where the region of 0.1 to $10 \mathrm{PHz}$ may be named petahertz radiation in analogy to the comparable region of terahertz [45]. This may extend the well-established technologies of electromagnetic waves. There, the visible light and with some constraints extended to the UVA is of special technological interest because the very high frequencies just below the problematic ionizing radiation allow comparably hazard-free [46] handling and unproblematic interaction with materials. Many feature of radio waves were found in the region of petahertz radiation because the dimensions of half a micron in the visible are still macroscopic (large and classical), however, chromophores as molecular resonators are microscopic (small) where quantum mechanics have to be considered. Special features can be established with the interaction of two or more chromophores where absorption spectra may be altered by exciton interactions, pathways for energy transport opened by FRET and special optical properties established by means of molecular metamaterials. As a consequence, although many aspects such as carrying optical signals have to be solved, the handling of petahertz radiation with interacting components can be considered as the target for devices of the next-generation technology.

\section{Acknowledgements}

This work was supported by the Fonds der Chemischen Industrie.

\section{References}

1. Lange K, Löcherer KH (1992) Meinke Gundlach. Taschenbuch der Hochfrequenztechnik ( $5^{\text {th }}$ edn. $)$. Springer Verlag, Berlin, Germany.

2. Langhals H (2013) Chromophores for picoscale optical computers:Sattler K (edn.), Fundamentals of picoscience, Taylor \& Francis Inc. CRC Press Inc., Bosa Roca US.

3. Bergmann L, Schaefer Cl, Gobrecht H, Matossi F (1966) Lehrbuch der Experimentalphysik, Band III, Optik. $\left(4^{\text {th }}\right.$ edn. $)$.Walter de Gruyter\& Co., Berlin,Germany.

4. Dürr H, Bouas-Laurent H (1990) Photochromism. Molecules and systems. Series: Studies in Organic Chemistry, Elsevier, Amsterdam,Netherlands.

5. Wang X-D, Wolfbeis OS (2013) Fiber-Optic Chemical Sensors and Biosensors (2008-2012). Anal Chem 85: 487-508.

6. American Radio Relay League (2013) The ARRL handbook for radio communications 2013. ( $90^{\text {th }}$ edn.). The American Radio Relay League, Inc., Newington, CT, 06111-1494,USA

7. Heeger AJ, SariciftciNS, Namdas EB (2010) Semiconducting and metallic polymers. Oxford University Press, Oxford,England.

8. Zollinger H (1993) Color chemistry. Synthesis, properties and applications of organic dyes and pigments.( $3^{\text {rd }}$ edn.).Wiley, Weinheim, Germany.

9. Clar E (1964) Polycyclic Hydrocarbons. Academic Press, London, UK.

10. Langhals H (2008) Molecular devices. Chiral, bichromophoric silicones: Ordering principles in complex molecules. In F. Ganachaud, S. Boileau, B. Boury (edns.). Silicon based polymers, Springer, Berlin,Germany.

11. Langhals $\mathrm{H}$ (2005) Control of the interactions in multichromophores: Nove concepts. Perylene bisimides as components for larger functional units. Helv Chim Acta 88: 1309-1343.

12. Langhals $H$ (1995) cyclic carboxylic imide structures as structure elements of high stability. Novel developments in perylene dye chemistry. Heterocycles 40 : 477-500.

13. Langhals H, Demmig S, Potrawa T (1991) the relation between packing effects and solid state fluorescence of dyes. J Prakt Chem 333: 733-748.

14. Hunt GR, McCoy EF ,Ross IG (1962) Excited states of aromatic hydrocarbons: Pathways of internal conversion. Aust J Chem 15: 591-604.

15. Rothammel K (1976) Antennenbuch. (5 ${ }^{\text {th }}$ edn.). Telekosmos Verlag, Stuttgart, Germany.

16. Rüdenberg R (1908) Der Empfang elektrischer Wellen in der drahtlosen Telegraphie. Ann D Phys 330: 446-466.

17. Rüdenberg R (1908) Ann d Phys Leipzig 25: 466-500.

18. McCoy EF, Ross IG (1962) Electronic states of aromatic hydrocarbons: The Franck-Condon principle and geometries in excited states. Aust J Chem 15 573-590.

19. Langhals $H$ (2000) A re-examination of the line-shape of the electronic spectra of complex molecules in solution. Log-normal function versus Gaussian. Spectrochim Acta 56: 2207-2210.

20. Langhals $H$ (2002) UV-visible spectroscopy and the potential of fluorescent probes. In F. H. Frimmel, Refractory Organic Substances in the Environment, p. 200-214, Wiley-VCH, Weinheim, Germany.

21. Langhals $H$ (2002) The rapid identification of organic colorants by UV/Visspectroscopy. AnalBioanal Chem 374: 573-578.

22. Weiser G, Fuhs W, Hesse HJ (1980) Study of polariton resonances in a cyanine dye crystal. Chem Phys 52: 183-191.

23. Penelly RR, Eckhardt CJ (1976) Quasi-metallic reflection spectra of TCNQ single crystals. Chem Phys 12: 89-105.

24. Philpott MR (1973) Advances in chemical physics.(I. Prigogine, S. A. Rice edns.) 23:227, Academic Press, New York, USA

25. Schmelzer H (1976) Origin of bronzing in glossy black printing inks. Deutsche Farben-Zeitschrift 30: 277-278; ChemAbstr 85: 162017.

26. Schmelzer $H$ (1976) Cause of bronzing in tinted black printing inks. FATIPEC Congress 1976, 13: 572-574; ChemAbstr 86: 56822.

27. Davydov, AS (1848) Theory of absorption spectra of molecular crystals. Zhur Eksptl. i Teoret. Fiz. 18: 210-218; ChemAbstr 43: 24604

28. Davydow AS (1962) Theory of Molecular Excitations. Transl. Kasha H.Oppenheimer, Jr., M. McGraw-Hill, New York, USA.

29. Langhals H, Rauscher M, Strübe J, Kuck D (2008) Three orthogonal chromophores operating independently within the same molecule. J Org Chem 73: 1113-1116.

30. Kuck D (2006) Functionalized aromatics aligned with the three Cartesian axes: Extension of centropolyindane Pure Appl Chem 78: 749-775.

31. Clegg RM (2006) The history of FRET: From conceptions to the labours of birth. In Reviews in Fluorescence 2006. (C. D. Geddes, J. R. Lakowicz, edns.).Springer US, New York, USA.

32. Perrin JB (1925) Fluorescence et radiochimie Conseil de Chemie. ( $2^{\text {nd }}$ edn.) Solvay, Paris.

33. Förster T (1946) Energy migration and fluorescence. Naturwiss.33: 166-175

34. Förster T (1948) Intermolecular energy transference and fluorescence. AnnPhys2: 55-75.

35. Förster T (1949) Experiments on intermolecular transition of electron excitation energy. Z. Elektrochem. 53: 93-99; ChemAbstr43: 33629

36. Förster T (1949) Experimental and theoretical investigation of intermolecular transfer of electron activation energy. Zeitschr. Naturforsch. 4a: 321-327; ChemAbstr44: 43074

37. Langhals $\mathrm{H}$, Poxleitner S, Krotz O, Pust T, Walter A (2008) FRET in orthogonally arranged chromophores. Eur. J Org Chem 73: 4559-4562.

38. Langhals H, Esterbauer AJ, Walter A, Riedle E, Pugliesi I (2010) Förste 
Citation: Langhals H (2014) Handling Electromagnetic Radiation beyond Terahertz using Chromophores to Transition from Visible Light to Petahertz Technology. J Electr Electron Syst 3: 125. doi:10.4172/2332-0796.1000125

Page 8 of 8

resonant energy transfer in orthogonally arranged chromophores. J Am Chem Soc 132: 16777-16782.

39. Pugliesi I, Walter A, Langhals H, Riedle E (2011) Highly efficient energy transfer in a dyad with orthogonally arranged transition dipole moments: Beyond the Limits of Förster. In M. Chergui, D. Jonas, E. Riedle, R. W. Schoenlein, A Taylor (edn.). Ultrafast Phenomena XVII, Oxford University Press, Inc., New York, USA.

40. Nalbach P, Pugliesi I, Langhals H, Thorwart M (2012) Noise-induced Förster resonant energy transfer between orthogonal dipoles in photoexcited molecules. Physical ReviewLett108: 218302(1)-218302(5).

41. Liu N, Giessen H (2010) Coupling effects in optical metamaterials. AngewChemInt Ed 49: 9838-9852.
42. Pendry JB, Holden AJ, Robbins DJ, Stewart WJ (1999) IEEE Trans. Microwave Theory Tech. 47: 2075-2084.

43. Linden S, Enkrich C, Wegener M, Zhou JF, Koschny T et al.(2004) Magnetic Response of Metamaterials at 100 Terahertz. Sci 306: 1351-1353.

44. Langhals $\mathrm{H}$, Hofer $\mathrm{A}$ (2013) Chromophores arranged as magnetic meta atoms: Building blocks for molecular metamaterials. J Org Chem 78: 5889-5897.

45. Perenzoni M, Paul DJ, eds. (2014) Physics and applications of terahertz radiation.Springer Series in Optical Sciences 173, Heidelberg, Germany.

46. Fitzpatrick, TB (1974) Sunlight and man. Normal and abnormal photobiologic responses.University Press, Tokyo, Japan. 